Jochen Hoffmann / Juliana Raupp

\title{
Politische Personalisierung
}

Disziplinäre Zugänge und theoretische Folgerungen

\section{Personalisierung und Image: Probleme der Politischen KOMMUNIKATIONSFORSCHUNG}

"[...] what the person protects and defends and invests his feelings in is an idea about himself, and ideas are vulnerable not to facts and things but to communications." (Goffman 1967: 43). Folgt man dieser Annahme von Erving Goffman, sind das Selbstbild einer Person und die Fremdbilder, welche dieses Bild bestätigen oder eben "verletzen« können, selbstverständliches Thema der Kommunikationswissenschaft und damit auch der Politischen Kommunikationsforschung. Das trifft durchaus zu, aber auf eine sehr spezifische Weise. Die Perspektive auf Selbst- und Fremddarstellungen von Politikern, wie sie in der Politischen Kommunikationsforschung vorherrscht, werden wir im Folgenden herausarbeiten und problematisieren. In Auseinandersetzung mit diesem Verständnis sollen alternative Zugangsweisen diskutiert und deren theoretischer Nutzen eingeschätzt werden.

Die sozialwissenschaftliche Literatur zum (öffentlichen) Bild von Politikern beschäftigt sich meist mit der schlagwortartigen These einer Personalisierung der Politik. Folgende Definitionen machen deutlich, dass es zwar kein einheitliches Verständnis von Personalisierung gibt, bestimmte Aspekte aber sehr typisch sind für die Wahrnehmung innerhalb der Politischen Kommunikationsforschung:

- "Die Personalisierung der Politik bedeutet, dass die komplexe, von vielfältigen Motivationen und Einstellungen geprägte Wahlentscheidung reduziert wird auf die alternative Entscheidung zwischen den Spitzenkandidaten. Die Person des Spitzenkandidaten wird zum Deutungsmuster komplexer politischer Tatbestände.» (Kaltefleiter 1981: 296)

- "Darunter wird verstanden, dass politische Programme und Ziele aufs intensivste mit zentralen politischen Rolleninhabern in Verbindung gebracht, auf sie reduziert werden, und damit Politik langfristig auf Personen verkürzt wird.« (Kaase 1986: 365)

- Im umfassenden Sinne meint der Begriff Personalisierung, dass in der politischen Berichterstattung des Fernsehens auf die Präsentation von Politikern stärker Nachdruck gelegt wird als auf die eigentlichen politischen Fragen.« (Lass 1995: 9)

Dr. Jochen Hoffmann ist Oberassistent am Institut für Kommunikations- und Medienwissenschaft der Universität Bern. Dr. Juliana Raupp ist Professorin am Institut für Publizistik- und Kommunikationswissenschaft der Freien Universität Berlin.

Die Autoren danken Daniela Spranger (Universität Zürich), Adrian Steiner (Universität Zürich) und Michel Wenzler (Universität Bern) für kritische und konstruktive Anregungen, die in den Beitrag eingeflossen sind.

Publizistik, Heft 4, Dezember 2006, 51. Jahrgang, S. 456-478 
- "Mit Personalisierung wird die Eigenschaft von medialen Darstellungen bezeichnet, handelnde Menschen in den Vordergrund der Berichterstattung zu stellen.« (Scherer 1998: 698)

Die Definitionen verstehen Personalisierung vor allem als einen reduktionistischen Prozess, als eine Verkürzung des Politischen auf Kosten von Themen-, Problem- oder Sachbezügen. ${ }^{1}$ Damit verbunden ist meist eine kritische Bewertung. Klaus Kindelmann beschreibt diese Perspektive und ihre normative Bias wie folgt: "Eine Wahlentscheidung, die auf Kandidaten und deren personalen Charakteristika basiert, ist aus dieser Sicht irrational, anspruchslos und von kurzfristigen Einflüssen bestimmt. Den Wählern wird wenig politisches Interesse und Verständnis zugebilligt. Die Kandidaten seien nur attraktiv verpackte Waren, hergestellt vom ,Image-Maker', der die Öffentlichkeit manipuliert, indem er spezielle, ansprechende Charakterzüge hervorhebt." (Kindelmann 1994: 27)

Kindelmann zeigt mit dieser Aussage nicht nur auf, wie stark Personalisierung als Begriff normativ besetzt ist. Deutlich werden auch die typischen Untersuchungsebenen, auf denen politische Personalisierung analysiert wird. Die Rede ist vom »Image-Maker", von Öffentlichkeit und von Wählern. Unterscheidbar werden Politik-, Medienund Bevölkerungsebene:

- Politikebene: Personalisierung gilt als Merkmal einer Amerikanisierung von Wahlkämpfen. Festzustellen sei eine Fokussierung auf Spitzenkandidaten und eine tendenzielle Entkoppelung von Kandidat und Partei (vgl. Brettschneider 2002: 14-20). Die Personenorientierung dominiere in Wahlkämpfen über die Themenorientierung.

- Medienebene: Personalisierung gilt als Merkmal der sogenannten Medienlogik. Für die Nachrichtenwertforschung ist Personalisierung einer der wichtigsten Nachrichtenfaktoren. ${ }^{2}$ Vor allem das Aufkommen des Fernsehens und der damit verbundene Visualisierungsdruck werden für eine zunehmende Bedeutung von Personenbezügen in der Politikberichterstattung verantwortlich gemacht (vgl. Wilke 1998: 286-287; Bußkamp 2002: 20-29).

- Bevölkerungsebene: Personalisierung gilt als Merkmal der Wählerorientierung. Zusammen mit Partei- und Themenorientierung bildet Personenorientierung die klassische Trias des sozialpsychologischen Modells der Wahlforschung (vgl. Campbell u. a. 1960). Diskutiert wird die These, ob mit nachlassenden Wählerbindungen an Parteien die Personenorientierung als erklärende Variable für Wahlentscheidungen an Bedeutung gewinnt (vgl. Brettschneider 2002: 75-89).

Die Dreiteilung in Politik-, Medien- und Bevölkerungsebene hat sich ohne Zweifel bewährt, doch die gleichzeitige perspektivische Verengung auf Wahlkampfkommunikation lässt sich nur pragmatisch erklären, nicht aber theoretisch begründen (vgl. Marcin-

1 Problematisiert wird die Gegenüberstellung von Personen- und Sachbezug von Holtz-Bacha/Lessinger/Hettesheimer (1998: 240); ausführlich hierzu auch Brettschneider (2002).

2 Vgl. Wilke (1998: 288-289). Diese Forschungstradition kann erklären, warum outputlastige Medieninhaltsanalysen dominieren und Journalisten als Handlungsträger und ihre Fremddarstellungsstrategien kaum Gegenstand prozessorientierter Studien sind; vgl. aber mit Blick auf die journalistische Gesprächsführung in Politiker-Interviews: Tapper (1998). 
kowski 2005: 343-344). Auch die Unterscheidung in Partei-, Themen- und Kandidatenorientierung ist weiterführend, doch während die Wahlforschung schon längst die komplexen Wechselwirkungen zwischen den drei Dimensionen fokussiert (vgl. Brettschneider 2002: 45-59), klammert sich die Politische Kommunikationsforschung weiterhin an die triviale Logik eines Nullsummenspiels: Personalisierung gehe auf Kosten von Themen- und/oder Parteiorientierung (vgl. z. B. Oberreuter 2001).

Ähnliche pragmatische Grundannahmen liegen Imagestudien zugrunde. Der Begriff "Image ist in der Wahlforschung und Politischen Kommunikationsforschung noch weniger theoretisch konturiert als Personalisierung (vgl. als Überblick Brettschneider 2002: 134-144). Entsprechend werden Imagemerkmale in empirischen Untersuchungen vor allem induktiv gewonnen. Als primäre beobachtungsleitende Differenz dient meist die Unterscheidung in politiknahe Politikereigenschaften wie Fachkompetenz und politikferne Politikereigenschaften wie Familie oder Hobbys (vgl. z. B. Kepplinger 1997: 180-181). Auch diese Differenzierung ist weniger theoretisch fundiert als Ausdruck normativer Vorannahmen. Um es überspitzt auszudrücken: Personalisierung sei schon schlimm genug, aber wenn damit auch noch eine Privatisierung des Politischen einhergehe, müssten die Alarmglocken der Demokratie besonders laut läuten (vgl. z. B. Ross 1998). Ein solches Verständnis von Personalisierung und Politikerimages hat additive Forschungsdesigns zur Folge, wo Personenbezüge und Sachbezüge, politische und apolitische Imagemerkmale ausgezählt und einander gegenübergestellt werden.

Auf diese Weise ist eine Fülle empirischer Personalisierungs- und Imagestudien entstanden, deren Ergebnisse kaum miteinander verglichen werden können. Verbindendes Element sind lediglich pragmatisch angewandte Unterscheidungen, die begriffliche Unschärfen aufweisen und kaum eine Strukturierungsleistung für vor allem empiriegeleitet gewonnene Kategorien erbringen können. Man wird konfrontiert mit additiven Listen von Variablen (vgl. zuletzt Kepplinger/Maurer 2005: 64-71), die in fast jeder Studie anders definiert und abgegrenzt werden.

Festzustellen ist somit ein Defizit an Untersuchungsanlagen, die vom konkreten Forschungsgegenstand abstrahierbar sind und vergleichend eingesetzt werden könnten. Die geforderte Abstraktionsleistung kann nur eine Theorie erbringen. Es müsste eine Theorie sein, welche die implizite Normativität, pragmatische Additivität und idiographische Genügsamkeit vorhandener Studien zugunsten eines relationalen Ansatzes mit nomothetischem Erklärungsanspruch überwindet. Dieser Beitrag will einen ersten Schritt auf diesem Weg zurücklegen. Ziel ist die systematische Darstellung theoretischer Perspektiven, die unterschiedliche Fachdisziplinen - Psychologie, Soziologie, Ökonomie und Politikwissenschaft - für die Personalisierungsdebatte bereithalten (Kapitel 2). Eine solche Reflektion ermöglicht die Dekonstruktion der oft impliziten Vorannahmen im dominanten Verständnis politischer Personalisierung. Sie dient darüber hinaus als Grundlage für unsere eigene theoretische Positionierung (Kapitel 3). Wir werden Vorschläge für eine Rekonstruktion zentraler Relationen machen und damit erste Schritte auf dem Weg zu einer kommunikationswissenschaftlich fundierten, interdisziplinären Theorie politischer Personalisierung zurücklegen. 


\section{Theoretische Perspektiven KOMMUNiKationswissensCHAFtLiCHER NACHBARDISZIPLINEN}

\subsection{Psychologische Perspektiven}

Eine Personalisierung, welche die Politische Kommunikationsforschung oft als Simplifizierung des Politischen beklagt, kann die Psychologie mit Hilfe von Schematheorien in ihrer Notwendigkeit erklären. Eine Schematheorie »is an information processing model of perception and cognition which attempts to isolate the mechanisms by which people come to understand the complex social world in which they live" (Augoustinos/Walker 1995: 32). Schemata sind demnach kognitive Strukturen aus Erwartungen, Wissen und Vorstellungen, die Umweltinformationen filtern, organisieren und bewerten. Schematisierte Wahrnehmung als erlernte Vereinfachung schont die Ressourcen individueller Informationsverarbeitung, übernimmt eine notwendige Orientierungsfunktion und bestimmt Relevanzen. ${ }^{3}$

Images lassen sich als personenbezogene Schemata ${ }^{4}$ konzipieren, deren besondere Leistungsfähigkeit darin liegt, dass sie Umwelt nicht nur strukturieren, sondern darüber hinaus eine Sozialisationsfunktion übernehmen: Lernen durch sozialen Vergleich wird möglich. Personen können als Vorbilder oder als Feindbilder definiert werden und lösen adaptive oder adversative Lernprozesse aus. Images werden so zu sozialen Bezugspunkten und sorgen für kommunikative Anschlussfähigkeit innerhalb spezifischer Milieus. Erzeugt wird die Differenz von Ingroup und Outgroup (vgl. Augoustinos/Walker 1995: 109-113). Der Zusammenhalt beispielsweise innerhalb einer Partei kann erhöht werden, wenn es gelingt, den eigenen Parteiführer zum sozialen Vorbild zu stilisieren und zugleich über Feindbilder Abgrenzungen vorzunehmen. Zur Gewährleistung dieser Orientierungs-, Sozialisations- und letztlich Integrationsfunktion zeichnen sich imagebildende Wahrnehmungsprozesse vor allem durch folgende Merkmale aus:

- Sich widersprechende Eigenschaften werden über spezifische Selektionen und Umdeutungen aufgelöst, so dass ein konsistentes Image entsteht.

- Eine bestimmte Eigenschaft wird besonders stark betont. Sie überstrahlt andere Eigenschaften und führt zu deren Marginalisierung (Halo-Effekt).

- Eine Eigenschaft wird einer bestimmten Gruppe oder Kategorie zugeordnet und daraus auf das Vorhandensein weiterer Eigenschaften geschlossen, die damit nicht notwendigerweise etwas zu tun haben (z. B. werden Brillenträgern höhere Intelligenz, aber geringere handwerkliche Fähigkeiten zugeschrieben).

Images als Selbst- und Fremdbilder werden zu handlungsleitenden Schemata, die es dem Individuum ermöglichen, in das soziale Leben hineinzufinden und sich in ihm zurechtzufinden. Damit schlagen psychologische Schematheorien zugleich eine Brücke zu den Sozialwissenschaften. Schemata sind auch »die kognitive Lösung des Sozialproblems, indem sie individuelles Agieren mit den Erwartungen anderer und mit sozialen Normen verknüpfen«(Vowe 1994: 444).

3 Vgl. zum Forschungsstand Augoustinos/Walker (1995: 32-59). Kommunikationswissenschaftliche Bezüge stellen Brosius (1991) und Scheufele (2003: 13-18) her.

4 Vgl. Cantor/Mischel (1979). Lau (1986) differenziert mit Bezug auf Politische Kommunikation in Wahlkämpfen »Groups«-, »Issues«-, "Candidate Personality«- und »Party«-Schemata. 
Das komplexe Wechselspiel von personaler Selbst- und Fremddarstellung wird nur selten als Untersuchungsgegenstand betrachtet (vgl. Mummendey 1995: 116). Für die Politische Kommunikationsforschung interessant sind vor allem psychologische Studien zu personalen Selbstdarstellungen. Die sogenannte Impression Management-Forschung (vgl. Mummendey ${ }^{2} 2002$ ) kann das vorherrschende Interesse an (massenmedialen) Fremddarstellungen von Politikern sinnvoll ergänzen. Es überrascht nicht, dass eine der wenigen Studien, die politische Selbstdarstellungsstrategien erforscht, von einer Psychologin vorgelegt wurde. Astrid Schütz (1992) unterscheidet zwischen assertiven, offensiven und defensiven Strategien. Als assertiv bezeichnet sie die positive Darstellung eigener Fähigkeiten, als offensiv die Kritik an Akteuren, die das eigene Selbstbild in Frage stellen, und als defensiv das Beschönigen, Leugnen und Rechtfertigen zur Abwehr negativer Fremdbilder. Untersuchungen zur Wirkung solcher Strategien legen Politikern ein gemischtes Vorgehen nahe: Stärken betonen, kleinere Fehler eingestehen, schwerwiegende Kritik zurückweisen und höchstens moderate Angriffe auf die Gegner (vgl. Schütz 1999: 112-115).

Neben Schematheorien, die auf einer hohen Abstraktionsebene imagebildende Informationsverarbeitungsprozesse erklären können, und der Impression Management-Forschung lohnt sich zum Dritten ein Blick auf persönlichkeitspsychologische Führungstheorien. Sie können die Frage beantworten, warum sich Spitzenpolitiker überhaupt dem Druck einer permanenten öffentlichen Beachtung und Bewertung aussetzen. Warum stellen sie sich als Resonanzfläche für soziale Erwartungen zur Verfügung?

Aus dem Blickwinkel von Führungstheorien kann die These vertreten werden, dass es vor allem die Persönlichkeitsstruktur des Narzissten ist, die derartige Neigungen fördert (vgl. Kets de Vries/Miller 1995). Narzissmus ist ein übermäßiges Verliebtsein in das Selbst. Eine solche Selbstliebe ist in Grenzen zweifellos funktional. Wenn sie pathologisch wird, äußert sie sich bei Narzissten in "Größenphantasien, Minderwertigkeitsgefühlen und übermäßigem Angewiesensein auf Bewunderung und Bestätigung durch andere. Sie haben wenig Einfühlungsvermögen für ihre Mitmenschen, gehen ausbeuterische, gelegentlich parasitäre Beziehungen zu diesen ein und leiden stark unter Neid. Sie neigen ferner dazu, Ereignisse und Personen entweder als nur gut oder als nur schlecht wahrzunehmen.» (Winterhoff-Spurk 1999: 15)

Deutlich wird, dass der Narzisst gerade wegen seiner Fixierung auf sich selbst die kommunikative Bestätigung seiner Mitmenschen besonders dringend benötigt. Personalisierung, Konfliktorientierung und Polarisierung wären somit nicht Phänomene, die von außen - etwa im Zuge von Medialisierungsprozessen - einer im Grunde sachlichen und rationalen Politik aufgedrängt werden (vgl. z. B. Sarcinelli 1992: 56), sondern sie sind auch in den Persönlichkeitsstrukturen der handelnden Akteure selbst begründet. Die These würde somit lauten: In die Politik und auf die öffentliche Bühne treibt es, wer ein übersteigertes Bedürfnis nach Anerkennung und Bewunderung hat und infolgedessen die Welt in Gut und Böse einzuteilen fähig ist.

Damit kann zusammenfassend festgehalten werden, dass die psychologische Perspektive in dreifacher Hinsicht einen Beitrag zur Erforschung von Personalisierung und Images in der Politischen Kommunikation leisten kann:

- Schematheorien können politische Komplexität vereinfachende Politiker-Images als ein notwendiges Produkt von Informationsverarbeitungsprozessen erklären. Sie geben 
Hinweise auf Konstruktionsprinzipien von Images, die über die in der Politischen Kommunikationsforschung üblichen deskriptiven Merkmalslisten hinausgehen und zugleich die verbreitete kulturkritische Abwertung von politischer Personalisierung in Frage stellen.

- Impression Management-Theorien wenden sich der von der Politischen Kommunikationsforschung ebenfalls vernachlässigten Frage zu, mit Hilfe welcher Strategien Politiker eine angestrebte Fremdwahrnehmung der eigenen Person zu erreichen versuchen. Somit könnten Forschungsdesigns durch prozessuale Analysen der politischen Selbstdarstellung optimiert werden.

- Führungstheorien verweisen auf Persönlichkeitsmerkmale als erklärende Variable für Personalisierung. Auch diese Perspektive spielt in der Politischen Kommunikationsforschung fast keine Rolle. Die Erklärungsrichtung führt fast immer von der Makroauf die Mikroebene. Die Amerikanisierungsdebatte und ihre wichtigste Argumentationsfigur ist ein typisches Beispiel: Politische Personalisierung sei in Europa weniger ausgeprägt als in den USA, weil sich Merkmale von politischen Systemen und Mediensystemen stark unterscheiden würden. Mit einer solchen Flucht auf die Makroebene wird die Rekursivität von individuellen Dispositionen und sozialen Strukturen ausgeblendet.

\subsection{Soziologische Perspektiven}

"Face is an image of self delineated in terms of approved social attributes [...]." (Goffman 1967: 5) Den Perspektivenwechsel von der Psychologie zur Soziologie bringt diese Aussage von Erving Goffman deutlich zum Ausdruck. Es ist der Perspektivenwechsel vom Individuum, seiner Informationsverarbeitung und seinen Persönlichkeitsmerkmalen zum sozialen Leben mit seinen Erwartungen und Normen.

Typisch für eine eher sozialdeterministische Deutung der Relation von Personalität und Gesellschaft sind konventionelle soziologische Rollentheorien. ${ }^{5}$ Eine Rolle gilt als Bündel normativer Verhaltenserwartungen. Dem liegt das Bild des "homo sociologicus« zugrunde: Der Mensch gilt als »als Träger sozial vorgeformter Rollen« (Dahrendorf 1977: 20). Sowohl in der konflikttheoretischen Variante von Ralf Dahrendorf wie auch in der strukturfunktionalistischen Perspektive von Talcott Parsons (1951: 201-325) verbindet sich mit einem solchen normativen Menschenbild eine »Oversocialized Conception of Man" (Wrong 1961), die vielfach kritisiert worden ist: Der Idee des Role-Taking sei die Idee des Role-Making an die Seite zu stellen (vgl. Turner 1962). In der Tradition des Symbolischen Interaktionismus (vgl. Mead 1995; Blumer 1969) muss die "Kreativität des Handelns" (Joas 1992) von Individuen theoretisch stärkere Berücksichtigung finden.

Diese Perspektive hat nicht zuletzt durch die These einer Individualisierung der Gesellschaft (vgl. Beck/Beck-Gernsheim 2002; Kron 2000) Rückenwind bekommen. Rollentheoretische Konzepte sind mehr und mehr von identitätstheoretischen Ansätzen abgelöst worden. Das Spiel mit Identitäten und die Fragmentierung von Identitäten scheinen das strukturfunktionalistische Paradigma von Rollenhandeln als Bedingung für eine Integration der Gesellschaft in Frage zu stellen.

5 Vgl. den noch immer beispielhaften Überblick und die Kritik von Krappmann (1988: 97-131). 
Was folgt daraus für die Frage der Personalisierung in der Politischen Kommunikation? Wie sind Begriffe zueinander in Beziehung zu setzen? Muss Personalisierung mit Individualisierung und muss Image mit Identität gleichgesetzt werden? Das folgende klassische Identitätsverständnis von Erik H. Erikson deutet an, dass es gerade für die Kommunikationswissenschaft hilfreich sein kann, eine Unterscheidung von Image und Identität beizubehalten:

"Das bewusste Gefühl, eine persönliche Identität zu besitzen, beruht auf zwei gleichzeitigen Beobachtungen: der unmittelbaren Wahrnehmung der eigenen Gleichheit und Kontinuität in der Zeit und der damit verbundenen Wahrnehmung, dass auch andere diese Gleichheit und Kontinuität erkennen.« (Erikson 1966: 18)

Diese Deutung bestätigt einmal mehr die notwendige Verknüpfung von Selbst- und Fremdwahrnehmung, wie sie insbesondere der Symbolische Interaktionismus postuliert. Eine Unterscheidbarkeit von Image und Identität ergibt sich daraus nicht. Es würde in eine theoretische Sackgasse führen, Image eher als Fremdwahrnehmung und Identität eher als Selbstwahrnehmung zu konzipieren. Auch Identität bildet sich über Interaktionen, im Wechselspiel von Selbst- und Fremdwahrnehmungen. Der Unterschied entsteht vielmehr aus dem Bezug zur Zeit. Erst wenn das Selbst, wie von Erikson formuliert, seine "Gleichheit und Kontinuität in der Zeit« wahrnimmt, kann von Identität gesprochen werden. Mit anderen Worten: Identität ist biographisch verwurzelt.

Auf den Imagebegriff trifft dies nicht zu. Nach Günter Bentele bezeichnet Image »das vereinfachte, typisierte und in der Regel bewertete Vorstellungsbild, das sich über Eindrücke, Wahrnehmungen oder Denkprozesse von irgendetwas (Objekte, Personen, Sachverhalte, Organisationen) bildet« (Bentele 1998: 657). Jede Begegnung mit einem unbekannten Menschen ist bereits imagebildend, wir machen uns ein Bild von diesem Menschen. Jede Berichterstattung über einen Politiker transportiert ein Image von diesem Politiker. Auch wenn sich ein Image verfestigt hat, bleibt es stets stärker dem Druck aktueller Kommunikationen ausgesetzt, als es die Identität je sein könnte. Daraus folgt: Das Individuum spielt in der Moderne, sofern man diese Moderne auch als Mediengesellschaft verstehen will, keineswegs mit seinen Identitäten, sondern "nur" mit seinen Images. Weder flüchtige Begegnungen können identitätsprägend sein noch Aktualitäten medialer Sekundärerfahrung.

Bezogen auf Politische Kommunikation würde dies bedeuten: Ein Politiker, der in der Zeitung etwas über sich liest, will etwas über sein öffentliches Image erfahren. Er sucht nicht nach seiner Identität in den Massenmedien, und er sucht auch nicht nach seiner sozialen Rolle. Er kennt seine Rolle, er weiß z. B., dass er als Oppositionspolitiker in Debatten polemischer argumentieren darf als der Regierungschef. Was somit als relevanter Mehrwert personengebundener politischer Information für diesen Politiker bleibt, ist sein Image. Denn hier fehlt die Gewissheit der biographisch verwurzelten Identität, und es fehlen die Gewissheiten von sozial verfestigten Rollen. Deshalb ist es nützlich, wenn die Kommunikationswissenschaft am klassischen Verständnis von Rolle und Identität festhält, aber diese um den Begriff Image als eine begriffstheoretische Antwort auf gesellschaftliche Veränderungen ergänzt: Mit Image kann die zunehmende Bedeutung und Kontingenz medial vermittelter Selbst- und Fremddarstellungen erfasst werden.

Entsprechend ist das identitätsbildende Potenzial politischer Massenkommunikation 
gering zu veranschlagen. Personalisierung in den Medien wäre vorrangig als imagebildender Prozess zu untersuchen, während Individualisierung eher als Identitätenvielfalt zu deuten ist. Identität ist "näher" am Menschen, sie kann persönlichkeitspsychologisch und biographisch erklärt werden, Image dagegen lässt sich als kategorielle soziale Größe leichter vom Individuum abstrahieren.

Aufgrund dieser theoretischen Positionierung kann der Image-Begriff auch das paradoxe Phänomen verarbeiten, dass Individualität selbst als soziale Erwartung beobachtbar ist. Dies ist z. B. dann der Fall, wenn einem Politiker vorgeworfen wird, dass er sich immer nur medial selbst inszeniere und dadurch seine individuelle Authentizität auf der Strecke bleibe. Eingeführt wird ein Glaubwürdigkeitspostulat, und daraus ergibt sich die theoretische Notwendigkeit, Individualität nicht als Ausbruch aus Rollenzwängen zu idealisieren. Vielmehr kann sie selbst zu einem Rollenzwang werden, dem sich ein Politiker nicht entziehen kann: Als Imageträger beugt er sich der sozialen Erwartung, dass er sich nicht sozialen Erwartungen beugt. Er inszeniert die Nicht-Inszenierung der eigenen Person. Er verkleidet sein Image als Identität. Individualität und Identität als Antipoden zum klassischen Rollenhandeln werden so zu einem genuinen Charakterzug des postmodernen Homo Sociologicus, der nicht zuletzt vom Imagebegriff dialektisch erfasst werden kann.

Funktional ist inszenierte Individualität als soziale Erwartung, weil dadurch Handlungsfähigkeit und Verantwortlichkeit des Menschen dokumentierbar werden. In einer kultursoziologischen Makroperspektive lassen sich solche Prozesse als Mythologisierung beschreiben: »Die Erwartung, dass es Personen sind, die die Geschicke der Menschheit lenken, gehört [...] zu den großen, institutionalisierten Mythen' moderner Gesellschaften." (Wehner 1998: 321) Ein Mythos ist eine Geschichte, die ein geschichtliches Phänomen erklärt. Die Erklärung liegt nicht in spezifischen Strukturen, sondern historische Entwicklungen werden auf Menschen und ihr individuelles Handeln zurückgeführt. Derartige Mythologisierungsmuster sind auch für die Politische Kommunikation der Gegenwart unentbehrlich: „Kommunikatoren und Rezipienten erwarten, dass nicht anonyme Mächte oder undurchsichtige Strukturen für irgendwelche Ereignisse verantwortlich zu machen sind, sondern dass Politiker in wichtigen Positionen Entscheidungen treffen und dafür verantwortlich gemacht werden können.» (Wehner 1998: 321)

Personenzentrierte kulturelle Mythen übernehmen somit eine gesellschaftliche Funktion: Sie können uns die Welt als Folge des Handelns von Menschen erklären, sie können Verantwortung zuweisen, indem Images Menschen unterscheidbar machen. Auf diese Weise reduzieren sie Komplexität - eine notwendige Funktion, wie bereits psychologische Schematheorien gezeigt haben, die Personalisierung schließlich auch aus dem Blickwinkel soziologischer Systemtheorien erklärbar macht: Personale Attributionen, Images und Reputationszuweisungen ermöglichen kommunikative Anschlussfähigkeit in sozialen Systemen. Bezogen auf das politische System bedeutet dies: »Durch die Darstellung des Politischen als ein personenabhängiges Geschehen finden zwei füreinander intransparente Kommunikationsbereiche, nämlich das politische System und die Menge der Wähler, ein Niveau wechselseitiger Erwartungen und Berechenbarkeit.» (Wehner 1998: 324) Das Publikum wird von politischer Komplexität entlastet, und Politik wird von komplexen Ansprüchen des Publikums entlastet. Die Konstruktion von 
Politiker-Images als systemische Leistung besteht somit in der Ermöglichung und in der Begrenzung der Inklusion des Wählers in das politische System.

Als Fazit aus der soziologischen Betrachtung von Personalisierung und Image kann demnach festgehalten werden:

- Das systemtheoretische Paradigma der Komplexitätsreduktion in System-Umwelt-Beziehungen knüpft an schematheoretische Vorstellungen der Psychologie an und kann einmal mehr deutlich machen, dass Personalisierung als Basisphänomen menschlicher bzw. systemischer Informationsverarbeitung konzeptualisiert werden muss.

- Der Imagebegriff ist als soziologische Kategorie notwendig, weil er eine Lücke füllt zwischen dem Begriff der sozialen Rolle und der sozialen Identität. Er kann der Kontingenz von Selbst- und Fremddarstellungen gerecht werden, wie sie sich vor allem in den Aktualitäten (politischer) Massenkommunikation äußert. Image als theoretische Größe verarbeitet Rollenhandeln und ist offen gegenüber sozialen Erwartungen einer individualisierten Gesellschaft, die Unverwechselbarkeit und Authentizität einfordert.

\section{3 Ökonomische Perspektiven}

Im Gegensatz zu soziologischen Systemtheorien sind Akteure zentrale Bausteine für ökonomische Theorien. Die Welt wird gleichsam vom Individuum her erklärt. Entsprechend überrascht es nicht, dass der Begriff Image vor allem in der Marketingliteratur präsent ist. Auch "politisches Marketing« ist mittlerweile zu einer gängigen Wortverbindung geworden (vgl. z. B. Newman 1994; Vowe/Wolling 2000; Schneider 2004a), und entsprechend kann die Frage nach dem theoretischen Beitrag der Wirtschaftswissenschaften zur Analyse von Personalisierung und zu Imageanalysen in der Politischen Kommunikation gestellt werden.

Auf der Grundlage des Rational Choice-Paradigmas lassen sich Wähler und Politiker als Akteure konzipieren, die eine Tauschbeziehung eingehen, wobei jeder versucht, für sich das Beste herauszuholen. Die Akteure tauschen Wahlstimme gegen Wahlversprechen. Das Angebot der Politik, das Wahlversprechen, ist nach Jürgen Heinrich (1998: 335) ein "Vertrauensgut, ein Gut, dessen Qualität vor dem Kauf gar nicht und nach dem Kauf und Konsum nur sehr ungenau erkannt werden kann«. Aus diesem Grund wird es für den Politiker notwendig, »billige Ersatzindikatoren für den Ausweis von Qualität zu bieten. Solche Ersatzindikatoren sind Personen, deren Namen als Marke positioniert werden können." (Heinrich 1998: 336)

Vertrauen erweckende Politiker ersetzen somit als sogenannter Shortcut ${ }^{6}$ den Aufwand, den der Wähler benötigt, um die Qualität des politischen Output und die Zuschreibbarkeit von Verantwortung angemessen beurteilen zu können. Gleichwohl bleibt der theoretische Gehalt des Markenbegriffs im Vergleich zum Image unterkomplex. Denn Marken zeichnen sich zunächst nur durch einen Verweischarakter aus. So verweist die Automarke Volkswagen streng genommen auf den Hersteller Volkswagen

6 Vgl. Lupia (1994) und zu Personen als Shortcuts Lau/Redlawsk (2001: 954). 
und auf sonst nichts. Erst durch das Markenimage wird dieser Verweis mit Inhalten gefüllt, die dem Käufer etwas über die Qualität des Produkts aussagen.

Der Verweischarakter der Marke ist gleichwohl theoretisch und mit Blick auf Politische Kommunikation weiterführend. Denn damit wird eine Repräsentationsbeziehung konstituiert, die das Nullsummenspiel der Trias Personenorientierung versus Parteiorientierung versus Themenorientierung aufhebt. Politiker als Marken führen gerade nicht zur De-Instituonalisierung und Entsachlichung von Politik, sondern können als Repräsentanten die genau gegenteilige Funktion erfüllen. »Mehr« Personalisierung bedeutet nicht zwangsläufig "weniger" Themen- oder Parteiorientierung.

Dies ist ein theoretischer Gewinn, und dennoch bleibt der Markenbegriff riskant, weil er durch seinen Verweis auf etwas "anderes in erkenntnistheoretische Sackgassen führen kann. Die Versuchung von Ökonomen, zwischen Marke und »eigentlichem" Produkt zu unterscheiden, dürfte größer sein als die Neigung von Soziologen, die Differenz von Image und Identität als Wirklichkeitsgefälle zu konstruieren. Ein Wirklichkeitsgefälle ergibt sich jedoch weder aus der Differenz von Produkt und Marke noch aus der Differenz von Identität und Image. Diese kritischen Einwände sollten bedacht sein, wenn wir als Fazit der ökonomischen Perspektive festhalten:

- Das ökonomische Verständnis von Politik als ein Vertrauensgut macht die besondere Relevanz von Personalisierung für das Funktionieren politischer Märkte deutlich. Der Wähler vertraut eher konkreten Menschen als anonymen Organisationen. Damit kann nach der psychologischen und soziologischen auch die ökonomische Perspektive Personalisierung als unabdingbaren Mechanismus einer sozialen Komplexitätsreduktion deutlich machen.

- Politische Images übernehmen eine Marketing- oder genauer: Markierungsfunktion. Die Unvereinbarkeit und Additivität von Personen-, Themen- und Parteiorientierungen wird aufgehoben. Stattdessen konstituiert sich eine Repräsentationsbeziehung.

\subsection{Politologische Perspektiven}

Es wurde bereits darauf hingewiesen, dass eine Orientierung etwa des Wahlverhaltens an personalen Attributen von Kandidaten "unter normativen Gesichtspunkten als minderwertig angesehen" (Brettschneider 2002: 25) wird. Genährt wird diese Sichtweise insbesondere von politologisch argumentierenden Beiträgen zu Personalisierung und Politikerimages. Sie sind meist demokratietheoretisch ausgerichtet und zeigen die stärkste normative "Ladung«. Eingefordert wird eine inhaltliche Orientierung an politischen Themen und/oder die prozessuale Orientierung an demokratischen Verfahren und Institutionen.

Damit lassen sich zwei grundlegende Demokratiemodelle gegen Personalisierungstendenzen der Politik in Anschlag bringen: Zunächst ist das deliberative Modell zu nennen mit seinem Kommunikationsmodus der freien Deliberation. Er gilt als konstitutiv für eine demokratische Meinungs- und Willensbildung. Im "herrschaftsfreien Diskurs" müssen die Personen hinter die Argumente zurücktreten (vgl. Wilke 1998: 285-286). Dem gegenüber steht das liberale Modell mit seiner prozessualen und institutionalisierten Gewährleistung gerechter Interessenskompromisse. Auch hier fehlen Personen als 
Legitimationsbegründung. Demokratie, so könnte die Schlussfolgerung in Anlehnung an Idealtypen Max Webers (2002) lauten, dürfe sich nur als rationale Herrschaft, nicht aber als charismatische Herrschaft vollziehen.

Die demokratietheoretische Tendenz, Personalität aus der Sphäre des Politischen auszugliedern, ${ }^{7}$ geht einher mit der Konstruktion einer Differenz von Öffentlichkeit und Privatheit. Öffentlichkeit gilt im deliberativen Modell als Ort der vernunftorientierten Kommunikation und im liberalen Modell als notwendiges Verfahrenselement der Demokratie. Außerhalb der Öffentlichkeit darf demnach keine Politik stattfinden. Es muss - positiv formuliert - einen vor politischem Zugriff geschützten privaten Bereich geben.

Vor diesem Hintergrund ist es keine Überraschung, dass das Lamentieren über zu viel Personalisierung in der Politik meist mit der Klage über eine Privatisierung des Politischen einhergeht. Richard Sennett bringt die kritische Deutung des Zusammenhangs zwischen Personalität und »Privatismus" wie folgt auf den Punkt: „Das Selbst wurde zum Grundprinzip der Gesellschaft. Der Verfall der öffentlichen Sphäre, in der es nichtpersonale Bedeutung und ein nichtpersonales Handeln gab, setzte an diesem Punkt ein." (Sennett 1983: 381) Sennett beklagt diese Entwicklung als Psychologisierung von Herrschaftsstrukturen durch Personalisierung, er sieht sich einer "Tyrannei der Intimität" ausgesetzt.

Unterscheidungen von öffentlich und privat, von rationaler und charismatischer Herrschaft verweisen auf die Notwendigkeit einer Selbstbegrenzung der demokratischen Gesellschaft. Eine Volksgemeinschaft als totalitärer Führerstaat kennt solche Grenzen nicht. Gleichwohl kann die demokratietheoretisch begründbare Unterscheidung von privat und öfentlich, die das Personale entpolitisiert, in theoretische Sackgassen führen. Im antiken Griechenland ermöglichte die Unterscheidung von öffentlich und privat Sklaverei trotz Agora (vgl. Imhof 1998: 18-19), und nicht ohne Grund war ein Motto der Frauenbewegung: „Das Private ist politisch!« Wer davon ausgeht, dass Politik überall dort stattfindet, wo Herrschaft ausgeübt wird, muss keineswegs die Grenze von öffentlich und privat aufheben. Sie wird jedoch nicht gegenstandsbezogen gesetzt (vgl. Schneider 2004b: 158-168), sondern Öffentlichkeit wird als eine zivilgesellschaftliche Potenzialität flexibilisiert.

Letztlich spiegelt sich im Verhältnis von Öffentlichkeit und Privatheit das für jede Gesellschaft grundlegende Spannungsverhältnis von Gleichheit und Freiheit. Öffentlichkeit, die als Idealtypus Gleichheit verbürgt, kann Freiheit beschneiden, aber Freiheit wird zu Recht beschnitten, wenn sie der Privatmensch nutzt, um Ungleichheitsverhältnisse zu begründen. Die Privatisierung des Politischen mag auf diesem Hintergrund weiter unzulässig bleiben; eine Politisierung des Privaten wird dann jedoch zu einer demokratischen Notwendigkeit. Zum Ausdruck kommt dies in soziologischen Konzepten wie dem der life politics von Anthony Giddens (1991: 214-231) oder in der Subpolitik von Ulrich Beck (1993: 149-171) - von Beck explizit verstanden als Rückkehr der Individuen in die Gesellschaft. So erfährt mit der politischen Transformation des Privaten auch das Personale eine normative Aufwertung - mit Konsequenzen für die Politische Kommunikation: Die Öffentlichkeit hat das Recht, private Nebenbeschäftigungen ei-

7 Vgl. auch die Kritik von Brettschneider (2002: 206-220). 
nes Politikers zum Politikum zu machen; die Öffentlichkeit hat die Pflicht, einen Politiker zu skandalisieren, der sich vor der Kamera für Werte stark macht, die er im Privatleben mit Füßen tritt; die Öffentlichkeit hat die Aufgabe, aus der privaten Biographie eines Menschen auf politisch relevante Merkmale und Einstellungen zu schließen.

Als Schlussfolgerung aus politologischen Perspektiven auf Personalisierung und Politiker-Images kann demnach festgehalten werden:

- Die politologisch ausgerichtete Literatur zu Personalisierung und Politiker-Images weist mit Recht auf Risiken für eine Demokratie hin, wenn Personen zu zentralen Legitimationsquellen für das politische Gemeinwesen werden.

- Diese Perspektive bleibt jedoch oft einseitig und ist mit einer Abwertung von Personalisierung verbunden. Das Personale wird in vermeintlich unpolitische private Räume verbannt. Eine solche normative Perspektive ist Ausdruck einer rationalistischen Voreingenommenheit, die nicht differenziert zwischen wünschenswerten und nicht wünschenswerten, zwischen funktionalen und dysfunktionalen Folgen politischer Personalisierung.

- Es ist deshalb auch ein Problem, zwischen öffentlichen und politischen Imagemerkmalen einerseits und privatistischen und apolitischen Imagemerkmalen andererseits zu differenzieren. Images sind nicht nur wahrnehmungspsychologisch, sondern auch öffentlichkeitssoziologisch und demokratietheoretisch zunächst unteilbar. Differenzierungen müssen kontextbezogen auf der Ebene der Relevanzzuweisungen erfolgen: Das Private und Personale kann politisch werden.

\section{Relationen als Bausteine einer Theorie politischer PERSONALISIERUNG}

Vier disziplinäre Perspektiven auf die Phänomene politische Personalisierung und Politiker-Images haben wir analysiert. Zum einen sind theoretische Bezüge von Deutungsmustern sichtbar geworden, die den Personalisierungsdiskurs der Politischen Kommunikationsforschung dominieren. Zum anderen wurden bislang vernachlässigte Sichtweisen eingeführt, begriffliche Differenzierungen vorgeschlagen sowie interdisziplinäre Anschlussmöglichkeiten erarbeitet. Auf dieser Grundlage werden wir im nächsten Schritt zentrale Relationen diskutieren, die Bausteine einer Theorie politischer Personalisierung sein können und spezifische Schlussfolgerungen für empirische Forschungsdesigns ermöglichen.

\subsection{Die Relation von Politik, Medien und Bevölkerung}

Die dargestellten psychologischen und soziologischen Ansätze begreifen Imagebildungsprozesse als komplexes Wechselspiel von Selbst- und Fremdwahrnehmung. Analysen der Politischen Kommunikationsforschung zur Personalisierung beschäftigen sich vor allem mit der Fremdwahrnehmung von Politikern, wie sie zum einen in Medieninhaltsanalysen und zum anderen durch Bevölkerungsumfragen sichtbar wird. Werden Untersuchungen auf der Medien- und der Bevölkerungsebene aufeinander bezogen, entstehen Designs, wie sie aus der Agenda-Setting-Forschung bekannt sind. Nur steht nicht die Frage im Mittelpunkt, wie die Medienagenda die Bevölkerungsaganda beein- 
flusst, sondern wie Medienimages die Wahrnehmung von Politikern in der Bevölkerung prägen. Mittlerweile findet in der Agenda-Setting-Forschung das politische Agenda Building zunehmend Beachtung, und so könnte auch in Imagestudien die politische Selbstwahrnehmung stärker einbezogen werden. Denn dann wird tatsächlich der theoretisch weiterführende Vergleich von Selbst- und Fremdwahrnehmung erfasst, und man beschränkt sich nicht nur auf die Gegenüberstellung unterschiedlicher Fremdwahrnehmungen.

Eine Orientierung für die empirische Umsetzung bieten beispielsweise Input-Output-Analysen, wie sie vor allem in der PR-Forschung üblich sind. ${ }^{8}$ Die Kommunikationsanregung der Öffentlichkeitsarbeit ist in dieser Tradition jedoch meist als unabhängige Variable gesetzt. Mit Bezug auf Personalisierung wäre es genauso wichtig zu fragen, wie sich politische Selbstbilder durch die Fremdbilder der Medien oder die Bevölkerungswahrnehmung verändern. Inwieweit reagieren Politiker und ihre Berater mit Imagekonstruktionen auf mediale Fremdzuschreibungen? Um solche Fragen zu beantworten, sind diachrone Forschungsdesigns einzufordern, die prozessual Wechselwirkungen auf allen drei Untersuchungsebenen erfassen.

\subsection{Die Relation von Darsteller und Publikum}

Massenmedien und Bevölkerung gelten als die klassischen Publika für politische Selbstdarstellungen. Die Relation von Politik auf der einen Seite und Massenmedien sowie Bevölkerung auf der anderen Seite wird gleichgesetzt mit der Relation von Darstellern und Publika. Der blinde Fleck bei der Parallelisierung beider Relationen ergibt sich daraus, dass Politiker nicht nur Darsteller, sondern selbst auch Publikum sein können. Das Dreieck von Politik, Medien und Bevölkerung als erprobte Heuristik der Politischen Kommunikationsforschung ist damit nicht in Frage gestellt. Zu problematisieren ist aber die Fixierung auf eine medienorientierte kommunikative Bringschuld der Politik, die politische Akteure vorschnell auf die Darstellerrolle reduziert (vgl. Sarcinelli 1998: 13-15).

Diese Fixierung auf Politik als Darstellungspolitik hat zur Folge, dass empirische Studien weniger die Personalisierung der Politik als die Personalisierung der medien- und bevölkerungsorientierten Politikvermittlung messen. Entsprechend ist die Personalisierungsdebatte stark auf Wahlkampfkommunikation fixiert. Der Bereich der sogenannten Entscheidungspolitik ${ }^{9}$ fällt unter den Tisch. Es interessiert die politische Kommunikationsforschung nicht, welche Rolle Personalisierung und Images in politischen Entscheidungsprozessen spielen.

Erklärt werden kann dies mit der normativen Ausgrenzung des Personalen aus dem Politischen. Sie trägt dazu bei, strategische Imagekonstruktionen von Politikern vorrangig als einen politikfremden Tribut an die Medienlogik zu begreifen. Dabei ist es durchaus plausibel, dass mit der Transformation traditioneller korporatistischer Strukturen und dem Aufkommen von unübersichtlichen informalen Netzwerken, Kommissionen und Expertenrunden (vgl. Blumenthal 2003) der Faktor interpersonales Vertrauen und mit ihm die Fremdwahrnehmung individueller Akteure als entscheidungsrelevanter

8 Vgl. hierzu die klassische Studie von Baerns (1991) sowie als Überblick Raupp (2005).

9 Vgl. zur Unterscheidung von Darstellungs- und Entscheidungspolitik Sarcinelli (1994). 
Shortcut an Bedeutung gewonnen hat. Impression Management zielt nicht nur darauf, von Journalisten oder vom Wähler als kompetent eingestuft zu werden. Ebenso kann professionelle Kompetenz in entscheidungspolitischen Arenen als Produkt personengebundener Darstellungskompetenz analysiert werden.

Personalisierung als ein soziales Grundphänomen ist demnach mehr als ein medialer Nachrichtenfaktor, der für Politik "höchstens" auf der Ebene von Wahlkämpfen relevant werden kann. Imagebildungsprozesse wären ebenso in ihrer Kopplung mit politischen Systemlogiken zu analysieren. Darauf aufbauend wäre es dann z. B. möglich, politische und mediale Personalisierungsprozesse zu vergleichen und ihre Wechselwirkungen zu untersuchen. Unsere Forderung lautet demnach, Politik auch als Publikum von Politik zu begreifen.

\subsection{Die Relation von Darstellung und Dargestelltem}

Die Erweiterung potenzieller Publika für politische Selbstdarsteller verbietet eine weitere oft implizite Parallelisierung von Relationen in der Politischen Kommunikation: Die Beziehung zwischen Darstellungspolitik und Entscheidungspolitik ist nicht gleichzusetzen mit der Beziehung zwischen Darstellung und Dargestelltem. Der Vorstellung, Darstellungspolitik habe Entscheidungspolitik einem Publikum möglichst angemessen zu vermitteln, liegt eine Transportmetaphorik zugrunde, welche die Darstellungszwänge innerhalb der Politik ignoriert. Wir haben darauf hingewiesen, dass auch entscheidungspolitische Kommunikation immer als Kommunikation zwischen Selbstdarstellern und Publika analysiert werden kann. Insofern bleibt zwar die Operationalisierung von Impression Management als Akteurskompetenz von zentraler Bedeutung für die Analyse politischer Personalisierung, sie sollte jedoch nicht a priori als Medialisierungsphänomen gedeutet werden. Imagestudien sind verfehlt, die in ihren Merkmalslisten Darstellungskompetenzen ${ }^{10}$ gleichsam als Medienkompetenz neben den »eigentlichen" politikrelevanten Entscheidungskompetenzen der Akteure zu erheben versuchen.

Es ist somit eine doppelte Beziehung zu beachten: Zum einen kann sich Darstellungskompetenz je nach Publikum unterscheiden, sie ist nicht nur Medienkompetenz. Zum anderen bleibt Darstellungskompetenz als empirische Größe nicht interpretierbar, wenn keine spezifische Relation zum Dargestellten und damit zu anderen potenziellen Dimensionen des Images hergestellt wird.

Anstatt Darstellungskompetenz als eines von mehreren Imagemerkmalen zu isolieren, müsste es empirisch gelingen, die Thematisierung der Relation von Darstellung und Dargestellten als Metakommunikation zu identifizieren. Die Darstellung der Darstellungskompetenz von Politikern ist deshalb Metakommunikation, weil sie die Kompetenz des Akteurs vermittelt, andere Kompetenzen erfolgreich zu vermitteln (vgl. Schlenker/Weigold 1990; Hitzler 1996: 265-268). Findet sich in der Politikberichter-

10 Zur Erhebung von Darstellungskompetenzen werden unterschiedliche Begriffe und empirisch unterschiedlich weit gefasste Kategoriendefinitionen verwendet: Vgl. z. B. Graber (1972) (»Style«), Nimmo/Savage (1975) (»stylistic role»), Weaver u. a. (1981) (»appealing manner») Kepplinger u. a. (1986) (»Auftreten«), Miller/Wattenberg/Malanschuk (1986) (»charisma»), Kindelmann (1994) (»Ausstrahlung«), Lass (1995) (»Ausstrahlung, Charisma, Kommunikations-, Selbstdarstellungsfähigkeit, Ansehen«), Kepplinger (1997) (»Starqualitäten«), Brettschneider (2002) (»Ausstrahlung»), Schulz/Zeh (2003) ("Aussehen, Ausstrahlung«). 
stattung beispielsweise die Aussage "Er ist ein kompetenter Bildungspolitiker", dann wäre dies eine einfache Fremddarstellung. Die Aussage »Er stellt sich als ein kompetenter Bildungspolitiker dar« wäre dagegen Metakommunikation, insofern sie die Relation konstruiert, der sie selbst unterliegt.

Die Fremddarstellung der Kompetenz, eine Kompetenz darzustellen, hat als Metakommunikation vor allem Auswirkungen auf Reputationszuweisungen. Sie legt über die Konstruktion einer Differenz von Schein und Sein eine kritische Bewertung nahe. Glaubwürdigkeit und Vertrauen als Maßstäbe, auf deren besondere Bedeutung insbesondere die PR-Forschung verweist (vgl. Bentele/Seidenglanz 2005), sind dagegen nur erreichbar, wenn über eine positive Bewertung von Darstellungskompetenzen eine metakommunikative Konstruktion von Authentizität gelingt.

Die Darstellung der Darstellungskompetenz von Politikern wird so zu einer journalistischen Beobachtung zweiter Ordnung, die wiederum vom Wissenschaftler beobachtet werden kann. Eine solche Beobachtung dritter Ordnung kann jedoch nicht erreichen, wer empirisch Darstellungskompetenz additiv neben anderen Imagemerkmalen auflistet. Um die bloße Reproduktion der journalistischen Beobachterperspektive zu vermeiden, ist es notwendig, Darstellungskompetenz als Querschnittskategorie systematisch in Forschungsdesigns zu integrieren. Nur so kann die wissenschaftliche Darstellung der medialen Darstellung der politischen Darstellung gelingen.

\subsection{Die Relation von Image, Reputation und Prominenz}

Die Forderung, begriffliche Differenzierungen vorzunehmen für empirische Phänomene, die als Realtypen nur schwer unterscheidbar sind, gilt auch für das Verhältnis von Image zu Prominenz und Reputation. Prominent sind Personen, die in spezifischen Öffentlichkeiten eine besondere Aufmerksamkeit erlangen (vgl. Peters 1996), und Reputation wird Personen zugewiesen, die eine besondere Wertschätzung genießen (vgl. Eisenegger/Imhof 2004). Es geht somit um die Beachtung und Bewertung von Politikern, also um Variablen, die mit dem Image im Zusammenhang stehen, aber gleichwohl von ihm unterscheidbar bleiben müssen. So kann eine Person mehr oder weniger prominent sein, sie kann mehr oder weniger viel Reputation genießen, sie kann aber nicht mehr oder weniger viel Image haben. Image ist somit ein komplexes Merkmal, das im Gegensatz zu Beachtung und Bewertung nicht einfach auf einer Ordinalskala abgetragen werden kann. Nicht "Wie viel Image? lautet demnach die Frage, sondern: Aus welchen Komponenten setzt sich das öffentliche Image eines Politikers zusammen, und welche Konstruktionsprinzipien erklären, dass diese personenbezogenen Merkmalszuschreibungen sich zu einem konsistenten, handlungsleitenden Vorstellungsbild verdichten?

Prominenz kann in der politischen Massenkommunikation als Voraussetzung für die Entstehung und Verfestigung eines solchen Vorstellungsbilds gesehen werden, und zugleich beeinflusst öffentliche Aufmerksamkeit auch die Reputation. Empirische Studien weisen auf die Korrelation zwischen Bekanntheit und Reputation gesellschaftlicher Akteure hin (vgl. Merten 2000: 106). Gleichzeitig birgt größere Bekanntheit auch ein größeres Reputationsrisiko und kann im Kontext einer Skandalisierung zu Reputationsvernichtung führen (vgl. Eisenegger 2005: 68ff.). Entsprechend folgt für Forschungsdesigns: Beachtung als Prominenz und Bewertung als Reputation sind nicht 
dieselben Variablen und so können ihre Ausprägungen in unterschiedlichen Kombinationen auftreten.

Dasselbe gilt für Image und Reputation. Bentele (1998) ist zuzustimmen, wenn er Image als das »in der Regel bewertete Vorstellungsbild « (vgl. Kap. 2.2) bezeichnet, denn damit macht er gleichermaßen auf die theoretische Differenz wie auf den empirischen Zusammenhang von Image und Reputation aufmerksam. Entsprechend kann die analytische Leistung des Forschers darin bestehen, die Verknüpfung von Imagekomponente und Reputationszuweisung aufzulösen. Zum Beispiel: In einem Presseartikel wird ein Politiker als anpassungsfähig beschrieben. Eine beobachtete Empfänglichkeit des Politikers für externe Ansprüche (Imagekomponente) wird mit einem positiven Begriff (Reputationszuweisung) belegt, so dass ein bewertetes Vorstellungsbild im Sinne von Bentele entsteht. In welcher Kombination wertneutrale Imagekomponente und wertende Deutung miteinander vermengt werden, ist weitgehend kontingent. Derselbe Politiker kann von einer anderen Zeitung als opportunistisch kritisiert werden. Die Imagekomponente bleibt dieselbe, nur das wertende Vorzeichen ändert sich. Deshalb ist die Unterscheidung der Variablen Image und Reputation auf der theoretischen Ebene nützlich, aber auch im Hinblick auf die Kommunikationspraxis: So sollte der Politiker, wenn er als Opportunist gilt, sich nicht in einer Kehrtwende als durchsetzungsstark zu präsentieren versuchen. Ziel müsste vielmehr die Umdeutung des negativ bewerteten Opportunismus in die positiv bewertete Anpassungsfähigkeit sein.

Es ist somit vor allem die Reputationsvariable, welche die Entwicklung eines Images zu einem kontingenten und damit zu einem gleichermaßen beeinflussbaren wie riskanten Prozess macht. Man spricht von einem positiven oder negativen Image, nicht aber von einer positiven oder negativen Identität. Wer Image als binäre Legitimationsstruktur (vgl. Hoffmann 2003: 159-162) konzipiert und von Identität unterscheidet, gewinnt ein analytisches und relationales Konzept, dessen Erklärungspotenzial über dem pragmatisch-additiver Imagemerkmalslisten liegt, die oft unreflektiert die Vermengung von Image und Reputation reproduzieren.

\subsection{Die Relation von Person, Thema und Organisation}

Wie bereits erläutert, liegt ein additives Verständnis von Personalisierung auch der Trias Personenorientierung, Themenorientierung und Parteiorientierung zugrunde. Die Relationen dieser drei Dimensionen werden oft wie ein Nullsummenspiel behandelt. Als Schlussfolgerung aus der ökonomischen Perspektive haben wir stattdessen gefordert, Politiker-Images in ihrer Markierungsfunktion ernst zu nehmen. Ein Politiker kann für bestimmte Themen stehen, ein Politiker kann für eine bestimmte Partei oder für eine bestimmte Organisation stehen.

Markierungsfunktionen ermöglichen vor allem zwei Image-Merkmale: Kompetenz und Repräsentanz. Einem Politiker wird beispielsweise Kompetenz in der Bildungspolitik attestiert, oder er gilt als Repräsentant für eine bestimmte bildungspolitische Position. In beiden Fällen erfüllt die Person eine Markierungsfunktion für politische Inhalte.

Ähnlich lassen sich die Relationen der Imagemerkmale Kompetenz und Repräsentanz gegenüber Organisationen modellieren. So lässt sich einem Politiker neben bildungspolitischer Sachkompetenz auch die Kompetenz zuschreiben, ein Bildungsministerium erfolgreich zu führen, und natürlich sind auch Person und Organisation als Re- 
präsentationsbeziehung verknüpfbar. Ein Politiker gilt als Vertreter einer bestimmten Partei oder einer bestimmten Institution. ${ }^{11}$

Damit ergibt sich wieder keine additive, sondern eine relationale Struktur. Ein solcher theoretischer Zugang erübrigt die Frage, ob Politiker, Medien oder Publikum auf Kosten von irgendetwas anderem zu Personalisierungen neigen. ${ }^{12}$ Kompetenz- und Repräsentanzvariablen als zentrale Imagedimensionen lösen diesen Zwang zu Alternativen auf. Sie dekonstruieren die vor allem für das deliberative Modell relevante Alternative Personenorientierung versus Sachorientierung und die vor allem für das liberale Modell relevante Alternative Personenorientierung versus Organisationsorientierung. Das ermöglicht es, die Qualitäten von Beziehungen, die sich im Spannungsfeld von handelnden Akteuren und Strukturen vollziehen, als empirische Möglichkeiten zu beobachten.

\subsection{Die Relation von Politischem und Privatem}

„Wer aus privaten Schicksalsschlägen gestärkt hervortritt und neben der Ausübung des Berufs beispielsweise Kinder erzieht, möchte damit auch auf seine politischen Fähigkeiten verweisen. Hierdurch gelingt es dem Politiker, indirekt auf seinen politischen Arbeitsstil, seine Organisationsfähigkeit, Managerfähigkeit, Problemlösungskompetenz, Koordinationsfähigkeit, Belastbarkeit und auf seine Durchsetzungsstärke hinzuweisen. Die Privat- und Persönlichkeitssphäre des Politikers gewinnt hierdurch an Einfluss, um die für den Beruf des Politikers als wesentlich eingestuften Kompetenzen zu vermitteln.« (Bußkamp 2002: 208; vgl. auch Miller/Wattenberg/Malanchuk 1986)

Diese Interpretation von Heike Bußkamp macht noch einmal schlaglichtartig deutlich, dass es verfehlt wäre, Personalisierungs- und Privatisierungsprozesse per se als demokratieschädliche Entpolitisierungstendenzen zu interpretieren. Stattdessen kann eine "ganzheitliche" Sicht (vgl. Wehner 1998: 325) auf den Politiker auch als Privatmenschen zu einem legitimen Indikator für die Beurteilung seiner politischen Rolle werden. Möglich wird eine parasoziale Beziehung zwischen Wähler und Politiker (vgl. Gleich 1999) mit Glaubwürdigkeit und Authentizität als normativem Anspruch.

Die Frage, inwieweit Imagemerkmale politisch oder unpolitisch sind, lässt sich somit nicht "von außen « beantworten. Die politische Relevanz des Privaten ist Ergebnis einer sozialen Konstruktion, und somit sind es wieder empirische Daten, von denen eine Antwort verlangt werden muss. Diese Antwort kann je nach Kontext unterschiedlich ausfallen: Die Frage, ob jemand lieber Spaghetti oder Schweinshaxen mag, könnte für eine Karriere in der bayerischen Lokalpolitik durchaus von Bedeutung sein. Andere Merkmale berühren noch mehr Kontexte: Führungskompetenz etwa, die ein Kandidat in seinem bisherigen Leben unter Beweis gestellt hat. Eine solche Charaktereigenschaft wird weithin als politikrelevant anerkannt, und das, obwohl sie politikunspezifisch ist. Kaum einer wird bestreiten, dass Führungskompetenzen zuweilen auch Manager, Ver-

11 Darüber hinaus sind noch weitere Repräsentationsbeziehungen zwischen Personen und Strukturen denkbar, etwa mit Bezug auf bestimmte Schichten, Milieus, Weltanschauungen etc.; vgl. zur Relevanz sozialer Repräsentativität von Politikern in der Bevölkerungswahrnehmung Rebenstorf/Weßels (1989).

12 Brettschneider (2002: 209-210) kann hier nur teilweise gefolgt werden, wenn er »Themen oder Kandidaten?» als »falsch gestellte Frage« bezeichnet, eine apriorische Unterscheidung in Parteien- und Kandidatenorientierungen jedoch als "sachgerecht" empfindet. 
einsvorsitzende, Eltern und Hochschullehrer benötigen. Wer holzschnittartig zwischen unpolitischen Charaktereigenschaften und spezifischen Politikkompetenzen unterscheidet, verliert den Blick für rollenübergreifende Relevanzen von Imagekomponenten, die politisch werden können - oder auch nicht.

Weil somit einmal mehr Personalisierung als ein soziales Basisphänomen zu deuten ist, hilft es nicht, Politiker als Personen in einen politischen und in einen privaten Teil aufzutrennen, um anschließend die Beziehungslosigkeit zwischen beiden Dimensionen theoretisch, empirisch oder normativ zu konstruieren. Ganz im Gegenteil: Die Beziehungen selbst werden zum empirisch interessanten Untersuchungsgegenstand, und dafür benötigt die Forschung ein theoretisches Modell, das nicht ausgerechnet hier seine blinden Flecken produziert, sondern begrifflich und analytisch notwendige Instrumente für einen relationalen Zugang bereitstellt. Wie die Reputationsvariable auch wird Politikrelevanz zu einer Querschnittskategorie in Forschungsdesigns, die nicht a priori mit Imagekomponenten verknüpft werden sollte.

\section{SChlussfolgerungen: DekOnStruktions- Und REKONSTRUKTIONSVORSCHLÄGE}

Politische Personalisierung ist eine Anpassung an eine politikfremde Medienlogik, die zu einer Entsachlichung und Privatisierung des Politischen beiträgt - so die Annahme, von der viele Studien in der Politischen Kommunikationsforschung geprägt sind. Wir wollten durch den Rückgriff auf theoretische Perspektiven außerhalb der Kommunikationswissenschaft zunächst einen Beitrag zur Problematisierung der Problematisierung politischer Personalisierung leisten. Es sind vor allem psychologische, soziologische und ökonomische Perspektiven, die ein anderes Bild ergeben: Das Personale als eine soziale Konstruktion ist unentbehrlich, um sich die Welt verstehbar zu machen. Schematheorien zeigen dies aus einer wahrnehmungspsychologischen Perspektive. In der Soziologie machen vor allem systemtheoretische Ansätze auf das Personale als Mechanismus der Komplexitätsreduktion aufmerksam, der die Inklusion des politischen Publikums gleichermaßen ermöglicht und begrenzt. Ökonomische Theorien wiederum sprechen nicht von Inklusion, sondern von der Fähigkeit des Bürgers, auf politischen Märkten Entscheidungen zu treffen. Personen können hier zu partizipationsfördernden Shortcuts werden.

Diese "Ehrenrettung « des Personalen in der Politik ist eine erste Schlussfolgerung, die sich aus der Prüfung unterschiedlicher Theorieperspektiven ergibt. Darüber hinaus wurde deutlich, dass der häufig gebrauchte, aber theoretisch nur unzureichend gefasste Image-Begriff durchaus nützlich ist, um eine gerade für die Kommunikationswissenschaft relevante Lücke zwischen soziologischen Rollen- und Identitätstheorien zu schließen. Die Aktualitäten massenmedialer Kommunikationen erzeugen Kontingenzen, die in Widerspruch geraten zur Stabilität sowohl der traditionellen sozialdeterministischen Rolle wie auch der biographisch verankerten Identität. Es ist somit das Image und weniger die Rolle oder Identität eines Politikers, die auf öffentlichen (post) modernen Bühnen konstruiert wird, die dort Veränderungen unterliegt und auch beeinflussbar ist.

Diese begrifflichen Klärungen und ein Verständnis von Personalisierung als soziales 
Basisphänomen ermöglichen erste Schritte auf dem Weg zu einer Theorie politischer Personalisierung. Wenn eine Theorie ein System ist, das Beziehungen zwischen seinen Elementen erklärt, die wiederum soziale Phänomene repräsentieren, ist zunächst einmal »Aufräumarbeit« gefordert: In der Personalisierungsdebatte dominante, aber nicht plausible Relationen gilt es zu dekonstruieren. Die entstehende Tabula Rasa kann für eine Neukonstruktion von Relationen genutzt werden. Diese sind ergebnisoffen, können aber eine Ordnungsfunktion übernehmen für eine Theoriebildung, die vor allem empiriegeleitet zu leisten wäre.

Unsere Dekonstruktion setzt zunächst an der klassischen Dreiecksbeziehung der Politischen Kommunikationsforschung an: Politik, Medien und Bevölkerung. Alleine dadurch, dass sich die meisten Studien mit politischer Personalisierung auf der Medienund Bevölkerungsebene beschäftigen, entsteht der Eindruck einer durch die Medienlogik getriebenen Politik. Wer sich dagegen stärker politischen Akteuren zuwendet, kann von der psychologischen Impression Management-Forschung wie auch von Führungstheorien profitieren. Eine Aufwertung von politischen Einflussstrategien wie Persönlichkeitsstrukturen könnte die Schieflage in der Relation von Politik, Medien und Bevölkerung beheben.

Eine weitere Dekonstruktion stellt die in der Personalisierungsdebatte übliche Positionierung von Politik als Darstellungspolitik in Frage, die sich an Medien und Bevölkerung als Publika wendet. Publika finden sich ebenso innerhalb der Politik, und daraus folgt auch, dass das Verhältnis von politischer Darstellung und dargestellter Politik nicht mit Hilfe einer Transportmetaphorik erklärt werden kann.

$\mathrm{Zu}$ problematisieren ist demnach eine Parallelisierung und Vermengung von drei theoretisch relevanten Relationen: Die Relation von Politik, Medien und Bevölkerung ist etwas anderes als die Relation von Darstellern und Publika. Die Relation von Darstellung und Dargestelltem wiederum ist als eine sozial konstruierte Metakommunikation zu analysieren und nicht als Beziehung zwischen Politikvermittlung und »eigentlicher « Politik. Wer diese analytischen Differenzierungen vornimmt, vermeidet den theoretischen Kurzschluss, politische Personalisierung auf einen Aspekt der sogenannten Politikvermittlung zu reduzieren.

Auch beim Verhältnis von Image, Reputation und Prominenz ist vielfach eine fehlende Trennschärfe zu konstatieren. Ohne Zweifel stehen diese drei Variablen in einer komplexen Wechselwirkung. Doch diese lässt sich nur empirisch erforschen und in eine Personalisierungstheorie integrieren, wenn zunächst Image als Vorstellungsbild idealtypisch unterscheidbar wird von Reputation als Bewertung und Prominenz als Beachtung von Personen.

Eine weitere notwendige Dekonstruktion betrifft die aus der Wahlforschung bekannte Trias aus Personen-, Themen- und Parteiorientierung. Durch den additiven Charakter vieler Forschungsdesigns wird diese Relation faktisch auf ein Nullsummenspiel reduziert: Mehr Personenorientierung in der Politik bedeute weniger Themen- oder Parteiorientierung und umgekehrt. Hier ist es zunächst notwendig, die Fokussierung auf Wahlen (und damit einmal mehr Politikvermittlung) aufzugeben. Entsprechend ist die Parteiorientierung als Organisationsorientierung zu generalisieren. Darüber hinaus haben die theoretischen Grundlagen aus Psychologie, Soziologie und Ökonomie deutlich gemacht, dass die Dreiecksbeziehung von Person, Thema und Organisation kein Kon- 
kurrenzverhältnis ist, sondern transformatorischen Charakter hat. Es sind vor allem die Imagekomponenten Kompetenz und Repräsentanz, die diese Transformation ermöglichen, indem sie die Verknüpfung von Personen zu Themen und Organisationen herstellen.

Die letzte notwendige Dekonstruktion theoretischer Relationen knüpft an die Ausgangsbeobachtung an: die Problematisierung von Personalisierung als eine "gefährliche« Privatisierung des Politischen. Zu problematisieren ist stattdessen die Gegenüberstellung von Öffentlichkeit als Raum des Politischen versus Privatheit als Welt des Personalen. Es gilt, sowohl die apriorische Verknüpfung von Personalität und Privatheit als auch die Zementierung der Grenze von öffentlich und privat aufzulösen - dies deshalb, weil eine Veröffentlichung und damit Politisierung des Privaten nicht nur empirisch beobachtbar, sondern auch normativ begründbar ist.

Mit diesen Dekonstruktions- und Rekonstruktionsvorschlägen sind erste Schritte auf dem Weg zu einer Theorie politischer Personalisierung zurückgelegt. Es sind deshalb nur erste Schritte, weil die Personalisierungsdebatte in der Politischen Kommunikationsforschung gezeigt hat, dass weit reichende Thesen lediglich blinde Flecken produzieren, die empirisch nicht mehr aufgelöst werden können. Der Stab ist deshalb an die empirische Forschung weiterzureichen: Ihre Aufgabe wäre es, die Grundlagen für die weiteren Schritte in der Theoriearbeit zu legen.

\section{LITERATUR}

Augoustinos, Martha/Walker, Iain (1995): Social Cognition. An Integrated Introduction. London. Baerns, Barbara (21991): Öffentlichkeitsarbeit oder Journalismus? Zum Einfluss im Mediensystem. Köln.

Beck, Ulrich (1993): Die Erfindung des Politischen. Zu einer Theorie reflexiver Modernisierung. Frankfurt/Main.

Beck, Ulrich/Beck-Gernsheim, Elisabeth (Hrsg.) (52002): Riskante Freiheiten. Individualisierung in modernen Gesellschaften. Frankfurt/Main.

Bentele, Günter (1998): Image. In: Jarren, Otfried/Sarcinelli, Ulrich/Saxer, Ulrich (Hrsg.): Politische Kommunikation in der demokratischen Gesellschaft. Ein Handbuch mit Lexikonteil. Opladen, S. 657.

Bentele, Günter/Seidenglanz, René (2005): Vertrauen und Glaubwürdigkeit. In: Bentele, Günter/Fröhlich, Romy/Szyszka, Peter (Hrsg.):Handbuch der Public Relations. Wissenschaftliche Grundlagen und berufliches Handeln. Wiesbaden, S. 346-360.

Blumenthal, Julia von (2003): Auswanderung aus den Verfassungsinstitutionen. Kommissionen und Konsensrunden. In: Aus Politik und Zeitgeschichte, B 43, S. 9-15.

Blumer, Herbert (1969): Symbolic Interactionism. Perspective and Method. Englewood Cliffs.

Brettschneider, Frank (2002): Spitzenkandidaten und Wahlerfolg. Personalisierung - Kompetenz Parteien. Ein internationaler Vergleich. Wiesbaden.

Brosius, Hans-Bernd (1991): Schema-Theorie. Ein brauchbarer Ansatz der Wirkungsforschung? In: Publizistik, 36. Jg., S. 285-297.

Bußkamp, Heike (2002): Politiker im Fernsehtalk. Strategien der medialen Darstellung des Privatlebens von Politikprominenz. Wiesbaden.

Campbell, Angus u. a. (1960): The American Voter. New York.

Cantor, Nancy/Mischel, Walter (1979): Prototypes in Person Perception. In: Advances in Experimental Social Psychology, 12. Jg., S. 4-47.

Dahrendorf, Ralf (151977): Homo Sociologicus. Ein Versuch zur Geschichte, Bedeutung und Kritik der Kategorie der sozialen Rolle. Opladen.

Eisenegger, Mark (2005): Reputation in der Mediengesellschaft. Konstitution - Issues Monitoring Issues Management. Wiesbaden. 
Eisenegger, Mark/Imhof, Kurt (2004): Reputationsrisiken moderner Organisationen. In: Röttger, Ulrike (Hrsg.): Theorien der Public Relations. Grundlagen und Perspektiven der PR-Forschung. Wiesbaden, S. 235-256.

Erikson, Erik H. (21966): Ich-Entwicklung und geschichtlicher Wandel. In: Erikson, Erik H.: Identität und Lebenszyklus. Drei Aufsätze. Frankfurt/Main, S. 11-54.

Giddens, Anthony (1991): Modernity and Self-Identity. Self and Society in the Late Modern Age. Oxford.

Gleich, Uli (1999): Parasoziale Bindungen zu Politikern? In: Winterhoff-Spurk, Peter/Jäckel, Michael (Hrsg.): Politische Eliten in der Mediengesellschaft. Rekrutierung - Darstellung - Wirkung. München, S. 151-167.

Goffman, Erving (1967): On Face-Work. An Analysis of Ritual Elements in Social Interaction. In: Goffman, Erving: Interaction Ritual. Essays on Face-to-Face Behavior. New York, S. 5-45.

Graber, Doris A. (1972): Personal Qualities in Presidential Images. The Contribution of the Press. In: Midwest Journal of Political Science, 16. Jg., S. 46-76.

Heinrich, Jürgen (1998): "Ökonomische Theorie der Personalisierung des Politischen«. In: Imhof, Kurt/Schulz, Peter (Hrsg.): Die Veröffentlichung des Privaten - Die Privatisierung des Öffentlichen. Opladen, S. 332-339.

Hitzler, Ronald (1996): Die Produktion von Charisma: Zur Inszenierung von Politikern im Medienzeitalter. In: Imhof, Kurt/Schulz, Peter (Hrsg.): Politisches Raisonnement in der Informationsgesellschaft. Zürich, S. 265-288.

Hoffmann, Jochen (2003): Inszenierung und Interpenetration. Das Zusammenspiel von Eliten aus Politik und Journalismus. Wiesbaden.

Holtz-Bacha, Christina/Lessinger, Eva-Maria/Hettesheimer, Merle (1998): Personalisierung als Strategie der Wahlwerbung. In: Imhof, Kurt/Schulz, Peter (Hrsg.): Die Veröffentlichung des Privaten Die Privatisierung des Öffentlichen. Opladen, S. 240-250.

Imhof, Kurt (1998): Die Verankerung der Utopie herrschaftsemanzipierten Raisonnements im Dualismus Öffentlichkeit und Privatheit. Einführung. In: Imhof, Kurt/Schulz, Peter (Hrsg.): Die Veröffentlichung des Privaten - Die Privatisierung des Öffentlichen. Opladen, S. 15-24.

Joas, Hans (1992): Die Kreativität des Handelns. Frankfurt/Main.

Kaase, Max (1986): Massenkommunikation und politischer Prozess. In: Kaase, Max (Hrsg.): Politische Wissenschaft und politische Ordnung. Analysen zur Theorie und Empirie demokratischer Regierungsweise. Opladen, S. 357-374.

Kaltefleiter, Werner (1981): Personalisierung. In: Greiffenhagen, Martin/Greiffenhagen, Sylvia/Prätorius, Rainer (Hrsg.): Handwörterbuch zur politischen Kultur der Bundesrepublik Deutschland. Ein Lehr- und Nachschlagewerk. Opladen, S. 296-299.

Kepplinger, Hans Mathias u. a. (1986): Medientenor und Bevölkerungsmeinung. Eine empirische Studie zum Image Helmut Kohls. In: Kölner Zeitschrift für Soziologie und Sozialpsychologie, 38. Jg., S. 247-279.

Kepplinger, Hans Mathias (1997): Politiker als Stars. In: Faulstich, Werner/Korte, Helmut (Hrsg.): Der Star, Geschichte - Rezeption - Bedeutung. München, S. 176-194

Kepplinger, Hans Mathias/Maurer, Marcus (2005): Abschied vom rationalen Wähler. Warum Wahlen im Fernsehen entschieden werden. Freiburg i. Br.

Kets de Vries, Manfred F. R./Miller, Danny (21995): Narzissmus und Führung. In: Kieser, Alfred/Reber, Gerhard/Wunderer, Rolf (Hrsg.): Handwörterbuch der Führung. Stuttgart, S. 1609-1622.

Kindelmann, Klaus (1994): Kanzlerkandidaten in den Medien. Eine Analyse des Wahljahres 1990. Opladen.

Krappmann, Lothar (1988): Soziologische Dimensionen der Identität. Strukturelle Bedingungen für die Teilnahme an Interaktionsprozessen. Stuttgart (zuerst 1969).

Kron, Thomas (Hrsg.) (2000): Individualisierung und soziologische Theorie. Wiesbaden.

Lass, Jürgen (1995): Vorstellungsbilder über Kanzlerkandidaten. Zur Diskussion um die Personalisierung von Politik. Wiesbaden.

Lau, Richard R. (1986): Political Schemata, Candidate Evaluations and Voting Behavior. In: Lau, Richard R./Sears, David O. (Hrsg.): Political Cognition. London, S. 95-126.

Lau, Richard R./Redlawsk, David P. (2001): Advantages and Disadvantages of Cognitive Heuristics in Political Decision Making. In: American Journal of Political Science, 45. Jg., S. 951-971.

Lupia, Arthur (1994): Shortcuts versus Encyclopedias: Information and Voting Behavior in California Insurance Reform Elections. In: American Political Science Review, Nr. 88, S. 63-76. 
Marcinkowski, Frank (2005): Die »Medialisierbarkeit« politischer Institutionen. In: Rössler, Patrick/ Krotz, Friedrich (Hrsg.) (2005): Mythen der Mediengesellschaft - The Media Society and its Myths. Konstanz, S. 341-369.

Mead, George Herbert ( $\left.{ }^{10} 1995\right)$ : Geist, Identität und Gesellschaft aus der Sicht des Soziabehaviorismus. Frankfurt/Main (zuerst 1934).

Merten, Klaus (2000): Image. In: Merten, Klaus (Hrsg.): Das Handwörterbuch der PR. 1. Band. Frankfurt/Main, S. 104-106.

Miller, Arthur H./Wattenberg, Martin P./Malanschuk, Oksana (1986): Schematic Assessments of Presidential Candidates. In: American Political Science Review, Nr. 80, S. 521-540.

Mummendey, Hans D. (21995): Psychologie der Selbstdarstellung. Göttingen.

Mummendey, Hans D. (22002): Selbstdarstellungstheorie. In: Frey, Dieter/Irle, Martin (Hrsg.): Theorien der Sozialpsychologie, Band III, Motivations-, Selbst- und Informationsverarbeitungstheorien. Bern, S. 212-233.

Newman, Bruce I. (1994): The Marketing of the President: Political Marketing as Campaign Strategy. London.

Nimmo, Dan/Savage, Robert L. (1975): Image Typologies in a Senatorial Campaign: A Comparison of Forced versus Free Distribution Data. In: Political Methodology, 2. Jg., S. 293-318.

Oberreuter, Heinrich (2001): Image statt Inhalt? Möglichkeiten und Grenzen inszenierter Politik. In: Depenheuer, Otto (Hrsg.): Öffentlichkeit und Vertraulichkeit. Theorie und Praxis der Politischen Kommunikation. Opladen, S. 145-157.

Parsons, Talcott (1951): The Social System. Glencoe.

Peters, Birgit (1996): Prominenz. Eine soziologische Analyse ihrer Entstehung und Wirkung. Opladen.

Raupp, Juliana (2005): Determinationsthese. In: Bentele, Günter/Fröhlich, Romy/Szyszka, Peter (Hrsg.): Handbuch der Public Relations. Wissenschaftliche Grundlagen und berufliches Handeln. Wiesbaden, S. 192-208.

Rebenstorf, Hilke/Weßels, Bernhard (1989): Wie wünschen sich die Wähler ihre Abgeordneten? Ergebnisse einer repräsentativen Bevölkerungsumfrage zum Problem der sozialen Repräsentativität des Deutschen Bundestages. In: Zeitschrift für Parlamentsfragen, 20. Jg., S. 408-424.

Ross, Dieter (1998): Die Regression des Politischen. Die Massenmedien privatisieren die Öffentlichkeit. In: Imhof, Kurt/Schulz, Peter (Hrsg.): Die Veröffentlichung des Privaten - Die Privatisierung des Öffentlichen. Opladen, S. 149-156.

Sarcinelli, Ulrich (1992): Massenmedien und Politikvermittlung - Eine Problem- und Forschungsskizze. In: Wittkämper, Gerhard W. (Hrsg.): Medien und Politik. Darmstadt, S. 37-62.

Sarcinelli, Ulrich (1994): Mediale Politikdarstellung und politisches Handeln: analytische Anmerkungen zu einer notwendigerweise spannungsreichen Beziehung. In: Jarren, Otfried (Hrsg.): Politische Kommunikation in Hörfunk und Fernsehen. Elektronische Medien in der Bundesrepublik Deutschland. Opladen, S. 35-50.

Sarcinelli, Ulrich (1998): Politikvermittlung und Demokratie: Zum Wandel der politischen Kommunikationskultur. In: Sarcinelli, Ulrich (Hrsg.): Politikvermittlung und Demokratie in der Mediengesellschaft. Beiträge zur politischen Kommunikationskultur. Opladen, S. 11-23.

Scherer, Helmut (1998): Personalisierung. In: Jarren, Otfried/Sarcinelli, Ulrich/Saxer, Ulrich (Hrsg.): Politische Kommunikation in der demokratischen Gesellschaft. Ein Handbuch mit Lexikonteil. Opladen, S. 698-699.

Scheufele, Bertram 2003): Frames - Framing - Framing-Effekte. Theoretische und methodische Grundlegung des Framing-Ansatzes sowie empirische Befunde. Wiesbaden.

Schlenker, Barry H./Weigold, Michael F. (1990): Self-Consciousness and Self-Presentation: Being Autonomous versus Appearing Autonomous. In: Journal of Personality and Social Psychology, Nr. 59, S. 820-828.

Schneider, Helmut (2004a): Marken in der Politik. Erscheinungsformen, Relevanz, identitätsorientierte Führung und demokratietheoretische Reflexion. Wiesbaden.

Schneider, Ulrich F. (2004b): Der Januskopf der Prominenz. Zum ambivalenten Verhältnis von Privatheit und Öffentlichkeit. Wiesbaden.

Schütz, Astrid (1992): Selbstdarstellung von Politikern. Analyse von Wahlkampfauftritten. Weinheim.

Schütz, Astrid (1999): Selbstdarstellung in der Politik: Techniken und ihre Wirkung, in: Winterhoff-Spurk, Peter/Jäckel, Michael (Hrsg.): Politische Eliten in der Mediengesellschaft. Rekrutierung - Darstellung - Wirkung. München, S. 105-120. 
Schulz, Winfried/Zeh, Reimar (2003): Kanzler und Kanzlerkandidat in den Fernsehnachrichten. In: Holtz-Bacha, Christina (Hrsg.): Die Massenmedien im Wahlkampf. Die Bundestagswahl 2002. Wiesbaden, S. 57-81.

Sennett, Richard ( $\left.{ }^{4} 1983\right)$ : Verfall und Ende des öffentlichen Lebens. Die Tyrannei der Intimität. Frankfurt/Main.

Tapper, Christoph (1998): „Herr Bundeskanzler, wir bedanken uns sehr herzlich...«. Zum journalistischen Umgang mit Helmut Kohl und Rudolf Scharping im Bundestagswahlkampf 1994. In: Publizistik, 43. Jg., S. 22-39.

Turner, Ralph H. (1962): Role-Taking: Process versus Conformity. In: Rose, Arnold M. (Hrsg.): Human Behavior and Social Processes: An Interactionist Approach. London, S. 20-40.

Vowe, Gerhard (1994): Politische Kognition. Umrisse eines kognitionsorientierten Ansatzes für die Analyse politischen Handelns. In: Politische Vierteljahresschrift, 35. Jg., S. 423-447.

Vowe, Gerhard/Wolling, Jens (2000): Amerikanisierung des Wahlkampfs oder Politisches Marketing? Zur Entwicklung der politischen Kommunikation. In: Kamps, Klaus (Hrsg.): Trans-Atlantik, Trans-Portabel? Die Amerikanisierungsthese in der politischen Kommunikation. Opladen, S. 57-92.

Weaver, David A. u. a. (1981): Media Agenda-Setting in a Presidential Election. New York.

Weber, Max (52002): Wirtschaft und Gesellschaft. Grundriss der verstehenden Soziologie. Tübingen (zuerst 1922).

Wehner, Josef (1998): Öffentliche Meinung und Person - Zur Darstellung der Politik in den Medien. In: Imhof, Kurt/Schulz, Peter (Hrsg.): Die Veröffentlichung des Privaten - Die Privatisierung des Öffentlichen. Opladen, S. 318-331.

Wilke, Jürgen (1998): Analytische Dimensionen der Personalisierung des Politischen. In: Imhof, Kurt/Schulz, Peter (Hrsg.): Die Veröffentlichung des Privaten - Die Privatisierung des Öffentlichen. Opladen, S. 283-294.

Winterhoff-Spurk, Peter (1999): Politiker in der Mediengesellschaft: Eine Annäherung aus medienpsychologischer Perspektive. In: Winterhoff-Spurk, Peter/Jäckel, Michael (Hrsg.): Politische Eliten in der Mediengesellschaft. Rekrutierung - Darstellung - Wirkung. München, S. 9-30.

Wrong, Dennis H. (1961): The Oversocialized Conception of Man in Modern Sociology. In: American Sociological Review, 26. Jg., S. 183-193.

Korrespondenzanschriften: Dr. Jochen Hoffmann, Institut für Kommunikations- und Medienwissenschaft, Universität Bern, Lerchenweg 36, CH-3000 Bern 9

E-Mail: jochen.hoffmann@ikmb.unibe.ch

Prof. Dr. Juliana Raupp, Freie Universität Berlin, Institut für Publizistik- und Kommunikationswissenschaft, Malteserstraße 74-100, D-12249 Berlin

E-Mail: raupp@zedat.fu-berlin.de 